\title{
Erratum to: Nonlinear programming without a penalty function or a filter
}

\author{
N. I. M. Gould • Ph. L. Toint
}

Published online: 13 November 2011

(C) Springer and Mathematical Optimization Society 2011

\section{Erratum to: Math. Program. Ser. A (2010) 122:155-196 DOI 10.1007/s10107-008-0244-7}

This note reports a correction to the results obtained by [2], in which an error was unfortunately discovered during work with D. Robinson. The problem is in the proof of Lemma 3.10 of this reference, where it is claimed that Lemma 6.5.3 of [1] can be invoked to deduce that $\rho_{k}^{c} \geq \eta_{2}$, where $\rho_{k}^{c}$ is a specific ratio of achieved to predicted reduction is constraint violation and $\eta_{2}$ is a constant in $(0,1)$. As it turns out, the reasoning is only correct if the ratio $\left\|s_{k}\right\| /\left\|s_{k}^{R}\right\|$ is bounded above, where $s_{k}$ is the step at iteration $k$ and $s_{k}^{R}$ is its projection onto the range of the transposed Jacobian $J_{k}^{T}$.

Handling the case where this ratio is unbounded above turned out to be surprisingly complex. In particular, this required considering separately the cases where the tangential component of the step at iteration $k$ is large or small with respect to its normal component, where the meaning of "large" and "small" has to be defined very specifically. The convergence proof taking this distinction into account is therefore significantly more involved than the proof of [2], and cannot be presented in the form of a few corrections in the original text. The report [4] (including substantial contribution by D. Robinson) proposes a corrected version of [2], where other minor

The online version of the original article can be found under doi:10.1007/s10107-008-0244-7.

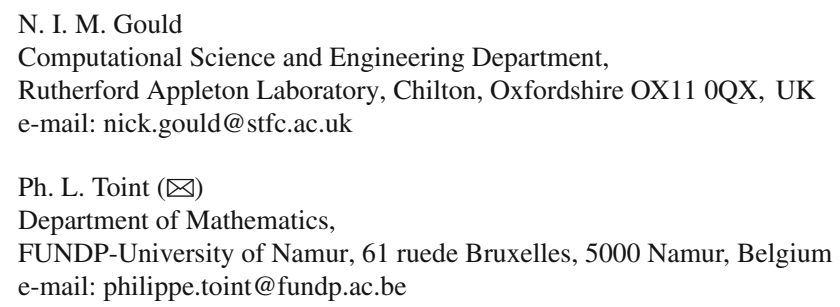


improvements and updates have also been introduced, including fixing a problematic case where it was possible to skip the normal step computation although the current iterate was close to the current infeasibility limit.

\section{References}

1. Conn, A.R., Gould, N.I.M., Toint, Ph.L.: Trust-Region Methods. MPS-SIAM Series on Optimization. SIAM, Philadelphia, USA (2000)

2. Gould, N.I.M., Toint, Ph.L.: Nonlinear programming without a penalty function or a filter. Math. Program. Ser. A 122(1), 155-196 (2010) [see also (Gould and Toint 2011)]

3. Gould, N.I.M., Toint, Ph.L.: Corrigendum: Nonlinear programming without a penalty function or a filter. Math. Program. Ser. A (2011, to appear)

4. Gould, N.I.M., Robinson, D.P., Toint, Ph.L.: Corrigendum: Nonlinear programming without a penalty function or a filter. Technical Report naXys-07-2011, Namur Centre for Complex Systems (naXys), FUNDP-University of Namur, Namur, Belgium (2011) 